\title{
Construction of layered $\mathrm{h}-\mathrm{BN} / \mathrm{TiO}_{2}$ hetero-structure and probing of the synergetic photocatalytic effect
}

\author{
Qun $\mathrm{Li}^{1}$, Xinmei Hou ${ }^{1 *}$, Zhi Fang ${ }^{1}$, Tao Yang ${ }^{1}$, Junhong $\mathrm{Chen}^{2}$, Xiangzhi Cui ${ }^{3}$, \\ Tongxiang Liang ${ }^{4}$ and Jianlin $\mathrm{Shi}^{3}$
}

\begin{abstract}
A novel layered hexagonal boron nitride/titanium dioxide (h- $\mathrm{BN} / \mathrm{TiO}_{2}$ ) composite photocatalyst has been constructed by anchoring $\mathrm{TiO}_{2}$ nanoflakes on the surface of $h$ BN flakes via a solvothermal method. The morphology and dispersion of $\mathrm{TiO}_{2}$ can be tuned by controlling the amount of flake h-BN. Benefiting from the unique hetero-structure, the photocatalytic performance of the obtained composite toward rhodamine $B(\mathrm{RhB})$ degradation is greatly enhanced, among which $12 \mathrm{wt} \% \mathrm{~h}-\mathrm{BN} / \mathrm{TiO}_{2}$ composites show 3.5 and 6.9 times higher degradation rate than the synthesized $\mathrm{TiO}_{2}$ and commercial $\mathrm{TiO}_{2}$ (P25), respectively, and an excellent cycling stability has also been obtained. Moreover, the first-principles calculation reveals the synergetic catalytic effect between $\mathrm{TiO}_{2}$ and $\mathrm{h}-\mathrm{BN}$ flake, which is found to be responsible for the significantly enhanced photocatalytic performance of $\mathrm{h}-\mathrm{BN} / \mathrm{TiO}_{2}$ composites.
\end{abstract}

Keywords: layered nanostructure, h-BN flake, $\mathrm{TiO}_{2}$ nanoflake, photocatalytic activity, dye degradation

\section{INTRODUCTION}

Photocatalytic degradation has increasingly become one of the effective approaches for solving environmental pollution by decomposing toxic complex organic pollutants into less toxic or even completely harmless molecules under sunlight or UV illumination [1]. Among the various semiconductor photocatalysts, titanium dioxide $\left(\mathrm{TiO}_{2}\right)$ has fostered considerable interest in organic dye degradation due to its high efficiency, low cost, nontoxicity and chemical stability [2,3]. However, owing to low utilization efficiency of sunlight (about 4\%) and the fast recombination of photogenerated electrons and holes, bare $\mathrm{TiO}_{2}$ exhibits low photocatalytic efficiency, which greatly limits its practical use [4]. Nowadays, two strategies are usually developed to enhance the photocatalytic activity. One is to decrease the size and design unique structure to promote the mobility and separation of photogenerated electrons and holes [5]. The other is to combine semiconductors with other favorable materials to construct semiconductor-based heterostructures and composite systems for visible-light-driven light harvesting systems [6,7]. To sum up, developing a composite system with a controllable structure by combining the above two advantages has profound meaning.

Hexagonal boron nitride (h-BN), with a two dimensional (2D) graphite-like structure, has opened up a new frontier in materials for the development of clean and renewable energy conversion and energy storage systems on account of high chemical stability, high thermal stability, low density, biocompatibility and environmental friendliness $[8,9]$. To our knowledge, h-BN is commonly excluded in photocatalysis and photovoltaic conversion applications due to its wide gap [10]. However, recent studies have proved the feasibility of h-BN as catalyst support materials to effectively enhance the photocatalytic properties of semiconductor materials because of the interactions between them, for instance, $\mathrm{SnO}_{2} / \mathrm{h}-\mathrm{BN}$ submicro-boxes (BNMB) [11], $\mathrm{BN} / \mathrm{Ag}_{2} \mathrm{CO}_{3}$ [12], h-BN/ $\mathrm{Bi}_{4} \mathrm{O}_{5} \mathrm{Br}_{2}$-layered microspheres [13], $\mathrm{CdS} / \mathrm{BN}$ nanosheets (BNNSs) [14], $\mathrm{BN} / \mathrm{AgBr}$ [15] and $\mathrm{BN} / \mathrm{Bi}_{4} \mathrm{O}_{5} \mathrm{I}_{2}$ [16]. The enhancement of photocatalytic propeties is generally ascribed to the suppressed recombination of photogenerated electrons and holes, extended excitation wavelength and increased amount of surface-adsorbed

\footnotetext{
${ }^{1}$ Collaborative Innovation Center of Steel Technology, University of Science and Technology Beijing, Beijing 100083, China

${ }^{2}$ School of Material Science and Technology, University of Science and Technology Beijing, Beijing 100083, China

${ }^{3}$ The State Key Laboratory of High Performance Ceramics and Superfine Microstructures, Shanghai Institute of Ceramics, Chinese Academy of Sciences, Shanghai 200050, China

${ }^{4}$ School of Materials Science and Engineering, Jiangxi University of Science and Technology, Ganzhou 341000, China

* Corresponding authors (emails: houxinmeiustb@ustb.edu.cn (Hou X); cuixz@mail.sic.ac.cn (Cui X))
} 
reactant [17]. However, the role of h-BN in the photocatalytic enhancement of the composites is still under debate. Furthermore, from the aforementioned BNsemiconductor composites, it can be found that the photocatalytic properties are largely determined by the morphology of the semiconductors and support materials $[18,19]$. In this work, layered $\mathrm{h}-\mathrm{BN} / \mathrm{TiO}_{2}$ composites with flake-on-flake hetero-structure are constructed and expected to improve photocatalytic activities. Multiple techniques including X-ray diffraction (XRD), scanning electron microscopy (SEM), transmission electron microscopy (TEM) and X-ray photoelectron spectroscopy (XPS) were used to characterize the obtained composites. The effect of the flake h-BN on the photocatalytic performance of the composites was investigated by degrading rhodamine $\mathrm{B}(\mathrm{RhB})$ under visible light irradiation. Moreover, the possible mechanism for the improved photocatalytic activity was discussed in detail by the firstprinciples calculation.

\section{EXPERIMENTAL SECTION}

\section{Preparation of flake $h-B N$}

All the chemicals used in this research were of analytical purity and without further purification prior to use. The synthesis of the $\mathrm{h}-\mathrm{BN}$ was reported in our previous paper [20]. Typically, the foamy precursor was prepared by low temperature combustion synthesis (LCS) method in air in a muffle furnace using $\mathrm{H}_{3} \mathrm{BO}_{3}, \mathrm{C}_{6} \mathrm{H}_{12} \mathrm{O}_{6} \cdot \mathrm{H}_{2} \mathrm{O}$, CO$\left(\mathrm{NH}_{2}\right)_{2}$ and $\mathrm{HNO}_{3}$ as raw materials. Then, h-BN was obtained by calcining the foamy precursor at $900^{\circ} \mathrm{C}$ for $3 \mathrm{~h}$ in flowing $\mathrm{NH}_{3}$. The residual carbon and $\mathrm{B}_{2} \mathrm{O}_{3}$ were removed by annealing in air and washing with hot water, respectively.

\section{Preparation of h-BN/TiO ${ }_{2}$ composites}

The h-BN/TiO ${ }_{2}$ composites with different contents of $h$ $\mathrm{BN}$ were prepared by a simple solvothermal process. Typically, $1 \mathrm{~mL} \mathrm{TiCl}_{3}$ and $1 \mathrm{~mL}$ deionized water were added into $30 \mathrm{~mL}$ of ethylene glycol under stirring to produce a light purple solution. Then, a certain amount of h-BN was suspended in the mixed solution, which was put into a 50-mL Teflon-lined stainless steel autoclave. The sealed autoclave was heated at $150^{\circ} \mathrm{C}$ for $12 \mathrm{~h}$ and then cooled naturally to room temperature. The asobtained products were centrifuged, washed with ethanol several times and dried at room temperature. Finally, the products were annealed at $350^{\circ} \mathrm{C}$ for $2 \mathrm{~h}$ to remove all organic species adsorbed on the composites and improve the crystallinity at the same time. The obtained samples were named as $X$ wt $\% \mathrm{~h}-\mathrm{BN} / \mathrm{TiO}_{2}$, where $X$ was the mass fraction of h-BN during the synthetic process. For comparison, $\mathrm{TiO}_{2}$ was synthesized by the similar method without addition of h-BN.

\section{Characterization}

The phase of the obtained samples was firstly examined by XRD (M21XVHF22, MAC Science, Yokohama, Japan) with a TTRIII diffractometer equipped with $\mathrm{Cu} \mathrm{Ka}$ radiation over a $2 \theta$ range from $10^{\circ}$ to $90^{\circ}$. The morphology and surface roughness of the obtained samples were observed by cold field emission SEM (FE-SEM, ZEISS SUPRATM 55, Germany). TEM (HITACHI H8100, Hitachi, Japan) was used to further investigate the microstructure. High resolution TEM (HRTEM, JEM 2010, Joel Ltd. Japan) was used to characterize the phase and crystal morphology of the products. The specific surface area was determined from the nitrogen adsorptiondesorption isotherm measured at $77 \mathrm{~K}$ on Quadrasorb SI-MP analyzer using Brunauer-Emmett-Teller (BET) model. The chemical states of the composites were determined by XPS. The optical properties of the samples were analyzed by UV-Vis spectrophotometer (UV2250, Shimadzu), in which $\mathrm{BaSO}_{4}$ was used as the reflectance standard material. Photoluminescence (PL) emission spectra were measured on a Hitachi F-7000 fluorescence spectrophotometer at an excitation wavelength of $361 \mathrm{~nm}$.

\section{Photoelectrochemical measurement}

The indium tin oxide (ITO) glass with an area of $1 \times$ $2 \mathrm{~cm}^{2}$ was washed ultrasonically in acetone and ethanol for a few minutes, respectively. The cleaned ITO glass was dried for the next modification. The h- $\mathrm{BN} / \mathrm{TiO}_{2}$ composites and polyvinylidene fluoride (PVDF) with the mass ratio of 95:5 were dispersed in $\mathrm{N}$-methyl-2-pyrrolidone (NMP) to form a stable suspension, and then the suspension was deposited on the ITO glass with an area of 1 $\times 1 \mathrm{~cm}^{2}$. Finally, the modified ITO glass was heated at $60^{\circ} \mathrm{C}$ overnight to evaporate the solvent for the photoelectrochemical measurement, which was tested in $0.2 \mathrm{~mol} \mathrm{~L}^{-1} \mathrm{Na}_{2} \mathrm{SO}_{4}$ solution on a CHI660D electrochemical working station consisting of an $\mathrm{Ag} / \mathrm{AgCl} \mathrm{re}-$ ference electrode, a platinum wire counter electrode, and a modified ITO glass working electrode. The photocurrent $v s$. irradiation time curve was recorded at $0 \mathrm{~V}$ under dark (20 s) and light (20 s), respectively. A light source used in the work was a $300 \mathrm{~W}$ Xe lamp with a $400 \mathrm{~nm}$ cut-off filter and the light intensity density was $100 \mathrm{~mW} \mathrm{~cm}^{-2}$. 


\section{Photocatalytic performance of the as-prepared sample}

Photocatalytic performance of the h- $\mathrm{BN} / \mathrm{TiO}_{2}$ composites was evaluated by irradiating $20 \mathrm{~mL} \mathrm{RhB}\left(10 \mathrm{mg} \mathrm{L}^{-1}\right)$ and phenol $\left(40 \mathrm{mg} \mathrm{L}^{-1}\right)$ in the presence of $0.75 \mathrm{~g} \mathrm{~L}^{-1} \mathrm{~h}-\mathrm{BN} /$ $\mathrm{TiO}_{2}$ under stirring. Before irradiation, the suspension was magnetically stirred for $30 \mathrm{~min}$ to achieve adsorption-desorption equilibrium. During the irradiation process, $5 \mathrm{~mL}$ of the suspension was collected and centrifuged to obtain supernatant. The concentration of $\mathrm{RhB}$ and phenol was examined at different time intervals by UV-vis spectrophotometer at 554 and $269 \mathrm{~nm}$, respectively. The degradation efficiency was calculated by the following expression [21]:

Efficiency $=\frac{C_{t}}{C_{0}}$,

where $C_{0}$ is the initial concentration of $\mathrm{RhB}$ and phenol solution; $C_{t}$ is the concentration at given test time.

\section{First-principles calculations}

All calculations were performed by density functional theory (DFT) using the Perdew-Burke-Ernzerhof (PBE) functional [22], as implemented in Vienna ab-initio Simulation Package (VASP) [23]. Electron-ion interactions were described by the projector-augmented wave (PAW) potentials with a kinetic energy cutoff of $450 \mathrm{eV}$ [24]. The valence electron configurations for $\mathrm{Ti}, \mathrm{O}, \mathrm{B}, \mathrm{N}, \mathrm{C}$, and $\mathrm{H}$ were $3 p^{6} 3 p^{2} 4 s^{2}, 2 s^{2} 2 p^{4}, 2 s^{2} 2 p^{1}, 2 s^{2} 2 p^{3}, 2 s^{2} 2 p^{2}$ and $1 s^{1}$, respectively. To correct the self-interaction error, the DFT $+\mathrm{U}$ method was adopted, where a Hubbard-type correction was applied on the $3 \mathrm{~d}$ orbitals of $\mathrm{Ti}$ and $2 \mathrm{p}$ orbitals of $\mathrm{O}$ [25]. Effective $U=4.2 \mathrm{eV}$ for $\mathrm{Ti} 3 \mathrm{~d}$ and $U=6.3 \mathrm{eV}$ for O $2 p$ orbitals were selected on the basis of previous work [26], which provided a correct description of the polaronic states of $\mathrm{TiO}_{2}$. The structure relaxation was stopped when the force on each atom was below $0.05 \mathrm{eV} / \AA$.

The anatase $\mathrm{TiO}_{2}$ (101) surface was modeled by $4 \times 1$ periodically repeated slabs and a four-layered model was used (Fig. S1a). A $20 \AA$ vacuum was added between slabs along the [101] direction to simulate the open surface. One layered h-BN was combined with $\mathrm{TiO}_{2}$ slab to model h-BN/TiO 2 composites (Fig. S1b). The lattice mismatch was smaller than $5 \%$ and the distance between these two slabs has been optimized. A Monkhorst-Pack grid of $6 \times 2 \times 1 k$ points mesh centered at the $\Gamma$ point was used for all DFT calculations. Electronic band structure and density of state (DOS) were calculated to analyze the contribution of h-BN to photocatalysis. Adsorption energies of organic molecules were applied to evaluate the ad- sorption capacity of photocatalysts and the charge-density difference was used to study the charge transfer between h-BN and $\mathrm{TiO}_{2}$. Electron effective masses $\left(m_{\mathrm{e}}{ }^{*}\right)$ and hole effective masses $\left(m_{\mathrm{h}}{ }^{*}\right)$ were calculated by the method of parabolic band fitting around the conduction band minimum (CBM) or the valence band maximum (VBM) according to the following equations:

$\left(m^{*}\right)_{i j}=\hbar^{2}\left[\frac{\partial \varepsilon_{n} \vec{k}}{\partial k_{i} k_{j}}\right]^{-1}, i, j=x, y, z$,

where $m^{*}$ is the effective mass of the charge carrier, $i$ and $j$ denote reciprocal components, $\varepsilon_{n} \vec{k}$ is the energy dispersion function of the $n$th band, $\vec{k}$ is the wave vector, and $\hbar$ represents the reduced Planck constant. For better fitting parabolic within the VBM and CBM regions, the nonself-consistent calculation for band structure was performed using $61 k$-points around $V B M$ or $C B M$ with a separation of $0.002 \AA^{-1}$.

In addition, the adsorption energies of $\mathrm{RhB}$ on photocatalysts were calculated to evaluate their adsorption ability. Because RhB molecule is so huge that the surface of slabs would be very large and the calculation would be quite time-consuming. Besides, de-ethylation of $\mathrm{RhB}$ is one of the most important steps during the degradation process. Two different parts of the RhB with ethyl groups were chosen as the adsorbed molecules.

\section{RESULTS AND DISCUSSION}

Fig. 1 shows the XRD patterns of $\mathrm{h}-\mathrm{BN}, \mathrm{TiO}_{2}$ and the annealed h-BN/TiO ${ }_{2}$ with different h-BN contents. As for $\mathrm{h}-\mathrm{BN}$, two broad diffraction peaks at $2 \theta=20^{\circ}-30^{\circ}$ and $40^{\circ}-45^{\circ}$ can be indexed to the (002) and (100) facets of $h$ $\mathrm{BN}$ (PDF 34-421), respectively. For $\mathrm{TiO}_{2}$, before calci-

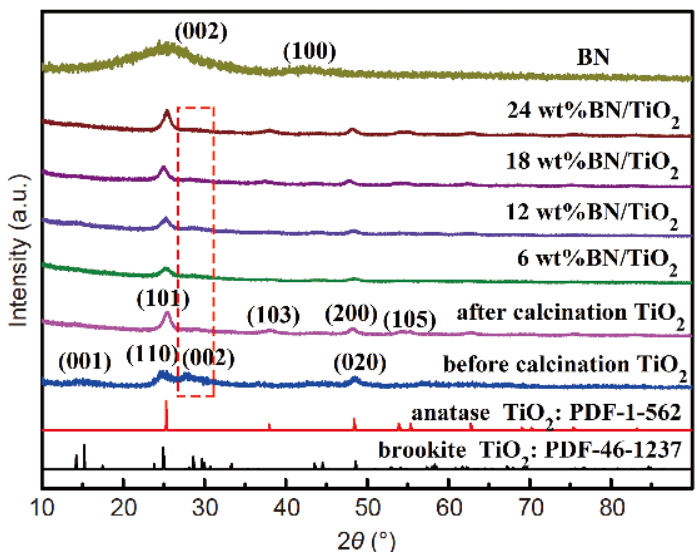

Figure 1 The $\mathrm{XRD}$ patterns of $\mathrm{h}-\mathrm{BN}, \mathrm{TiO}_{2}$ and $\mathrm{h}-\mathrm{BN} / \mathrm{TiO}_{2}$ samples with different contents of flake h-BN. 
nation, it can be clearly found that the peaks for (001), (110), (002) and (020) correspond to the $\mathrm{TiO}_{2}$ brookite (B) crystalline phase (PDF 46-1237) [27]. However, after calcination, the phase of the $\mathrm{TiO}_{2}$ gradually transforms from brookite to anatase crystalline phase (PDF 1-562). Interestingly, the phase transformation is not complete, which leads to the enhancement of the photocatalytic activity of $\mathrm{TiO}_{2}$ due to the existence of two crystal structures acting as semiconductor heterojunction [28]. After h-BN was introduced, all diffraction peaks of the obtained h- $\mathrm{BN} / \mathrm{TiO}_{2}$ composites can be clearly indexed to anatase $\mathrm{TiO}_{2}$ crystals except for the (002) small peak of the brookite phase (marked by the red line). And no distinct diffraction peaks of $\mathrm{h}-\mathrm{BN}$ can be observed due to dense $\mathrm{TiO}_{2}$ covering on its surface.

The effect of the flake h-BN content on the $\mathrm{TiO}_{2}$ microstructure was also investigated and shown in Fig. S2. Fig. S2a and b indicate that pure $\mathrm{TiO}_{2}$ assembled from 2D nanoflakes has a hierarchical microsphere structure with an average diameter of $200 \mathrm{~nm}$. After the flake h-BN is introduced, the structure of $\mathrm{TiO}_{2}$ gradually transforms from microsphere into randomly oriented nanoflakes and nano grasses structure has been formed with the increase of the addition of flake h-BN (Fig. S2c-j). The result reveals that the morphology and dispersion of $\mathrm{TiO}_{2}$ can be tuned by controlling the flake h-BN content. However, when the addition amount is excessive, the flake h-BN will stack and hinder the growth of $\mathrm{TiO}_{2}$ nanoflake, which leads to a light shielding effect and suppresses the generation of photogenerated electron-hole pairs. The optimized addition amount of flake $\mathrm{h}-\mathrm{BN}$ is $12 \mathrm{wt} \%$, and the $2 \mathrm{D} \mathrm{TiO}{ }_{2}$ nanoflake is uniformly attached on the surface of flake $\mathrm{h}-\mathrm{BN}$ to form a novel $2 \mathrm{D} / 2 \mathrm{D} \mathrm{h}-\mathrm{BN} / \mathrm{TiO}_{2}$ nanostructure composite.

In order to further investigate the growth mechanism of $\mathrm{TiO}_{2}$ on the surface of $\mathrm{h}-\mathrm{BN}$, the morphology of the obtained h-BN and $12 \mathrm{wt} \% \mathrm{~h}-\mathrm{BN} / \mathrm{TiO}_{2}$ was discussed in detail. From Fig. $2 a$ and $b$, it can be observed that the morphology of the h-BN possesses graphene-like flake structure, which is advantageous for the growth of $\mathrm{TiO}_{2}$ nanoflake and formation of $\mathrm{h}-\mathrm{BN} / \mathrm{TiO}_{2}$ heterojunctions. The HRTEM image in Fig. $2 \mathrm{c}$ shows four parallel fringes at the edge region of the $\mathrm{h}-\mathrm{BN}$ flake and the interlayer distance is $0.357 \mathrm{~nm}$, which is larger than the standard value of h-BN $(0.33-0.34 \mathrm{~nm})$ resulting from the existence of structure defects in the obtained flake h-BN [29]. When the flake h-BN is used to construct $2 \mathrm{D} / 2 \mathrm{D}$ h$\mathrm{BN} / \mathrm{TiO}_{2}$ composites, its surface becomes rough and the flake h-BN can hardly be observed due to the dense $\mathrm{TiO}_{2}$ nanoflakes covering on its surface as shown in Fig. $2 \mathrm{~d}$ and

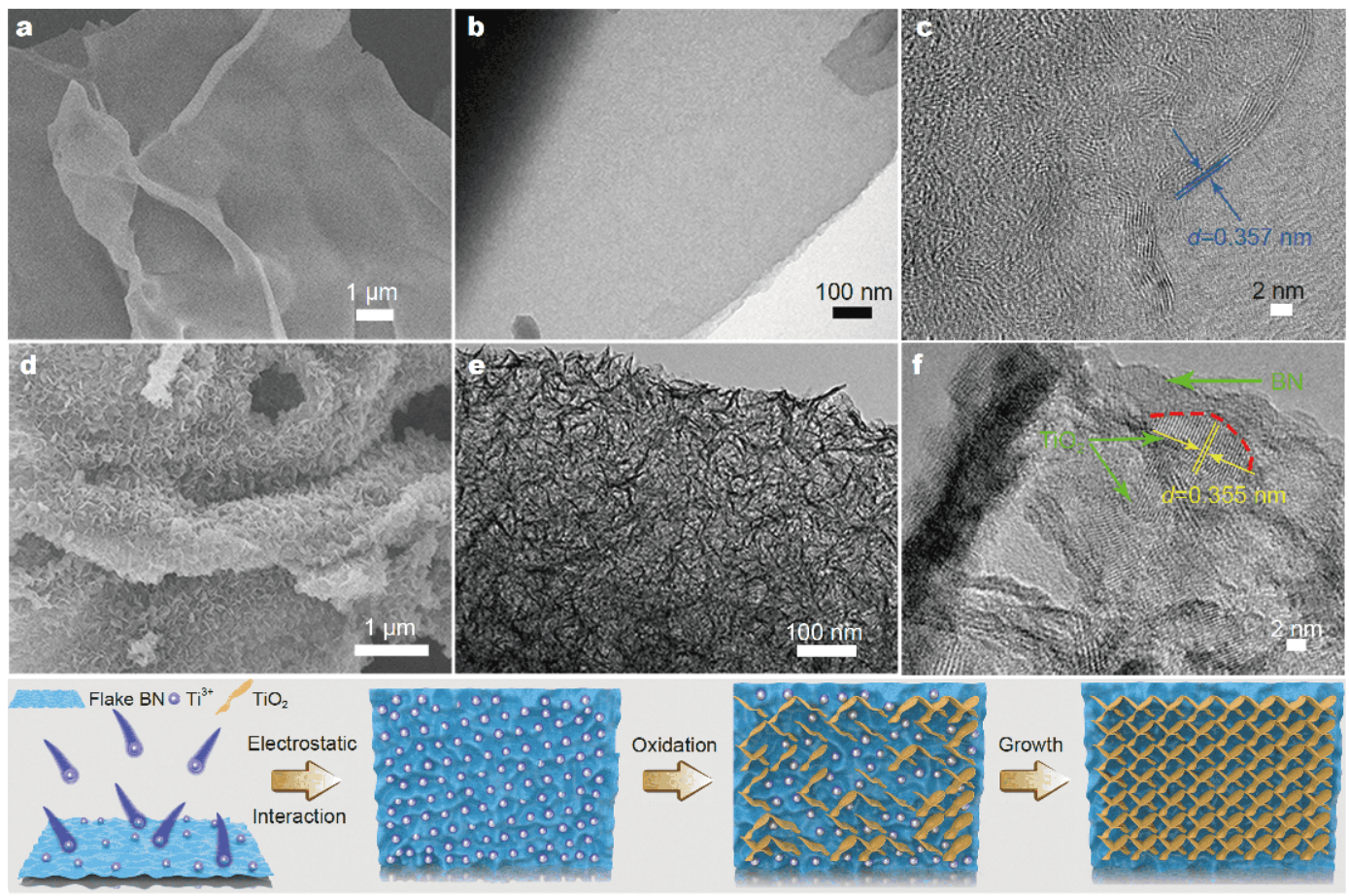

Figure 2 SEM (a) and TEM (b, c) images of the h-BN sample. SEM (d) and TEM (e, f) images of $12 \mathrm{wt} \% \mathrm{~h}-\mathrm{BN} / \mathrm{TiO}_{2}$. (g) Schematic illustration of the synthetic process and growth mechanism for $2 \mathrm{D} / 2 \mathrm{D}$ h- $\mathrm{BN} / \mathrm{TiO}_{2}$ composites. 
e. HRTEM image in Fig. $2 \mathrm{f}$ demonstrates the existence of interface structure of heterojunctions (marked by red lines) between $\mathrm{TiO}_{2}$ nanoflakes and flake h-BN. Due to the rich detects character of flake h-BN, only the (101) plane of the anatase phase $\mathrm{TiO}_{2}$ can be observed and the fringe spacing is $0.355 \mathrm{~nm}$. Based on the above results, the growth mechanism is proposed. It has been reported that the flake $\mathrm{h}-\mathrm{BN}$ has an overall negative surface charge at $\mathrm{pH}$ above 3.2 [20], which is important for the uniform growth of $\mathrm{TiO}_{2}$ nanoflakes on the surface of flake h-BN. As shown in Fig. 2g, when the flake of h-BN is added into the ethylene glycol solution containing $\mathrm{TiCl}_{3}, \mathrm{Ti}^{3+}$ will be adsorbed on the negatively charged surface of flake h-BN by electrostatic interaction. Then the $\mathrm{Ti}^{3+}$ is gradually oxidized to form $\mathrm{TiO}_{2}$ in the acidic solution during the prolonged solvothermal treatment. Meanwhile, due to the mechanical support of flake h-BN, the self-aggregated $\mathrm{TiO}_{2}$ microspheres will scatter into nanoflakes and grow on the h-BN surface.

The surface chemical composition of the $12 \mathrm{wt} \% \mathrm{BN} /$ $\mathrm{TiO}_{2}$ composite was also investigated by XPS analysis. Fig. 3a shows that Ti $2 p$ spectra consist of two major peaks and the binding energy of the Ti $2 \mathrm{p}_{3 / 2}$ peak shifts from 459.22 to $459.45 \mathrm{eV}$ after introduction of the flake $\mathrm{h}-\mathrm{BN}$, indicating the close interaction between flake h-BN and $\mathrm{TiO}_{2}$. In Fig. 3b, the $\mathrm{O}$ 1s spectra of $\mathrm{TiO}_{2}$ nanoflakes are identified to two peaks with the binding energy values at 530.02 and $531.64 \mathrm{eV}$, corresponding to $\mathrm{Ti}-\mathrm{O}$ and $\mathrm{O}-\mathrm{H}$ bonds, respectively. However, a new peak with the binding energy at $534.1 \mathrm{eV}$ corresponding to $\mathrm{Ti}-\mathrm{O}-\mathrm{B}$ bond appears in the $\mathrm{O}$ 1s spectra of $12 \mathrm{wt} \% \mathrm{BN} / \mathrm{TiO}_{2}$ besides the same two peaks as $\mathrm{TiO}_{2}$ [30]. Appearance of the new Ti-O-B bond further confirms the formation of heterojunctions between flake h- $\mathrm{BN}$ and $\mathrm{TiO}_{2}$ nanoflakes.

The $\mathrm{N}_{2}$ adsorption-desorption isotherms were conducted to determine the specific surface area (SSA) of the flake h-BN, pure $\mathrm{TiO}_{2}$ and $12 \mathrm{wt} \% \mathrm{~h}-\mathrm{BN} / \mathrm{TiO}_{2}$. The isotherm of the h-BN can be classified as type IV isotherm with type $\mathrm{H} 4$ hysteresis loop according to the IUPAC nomenclature [31], which reveals a predominant mesoporous structure (Fig. S3a). The BET model gives an SSA of $936 \mathrm{~m}^{2} \mathrm{~g}^{-1}$, and Barrett-Joyner-Halenda (BJH) calculation gives the total pore volume of $0.705 \mathrm{~cm}^{3} \mathrm{~g}^{-1}$ for $\mathrm{h}$ $\mathrm{BN}$, which is favor of the species adsorption. As shown in Fig. S3b, the SSA and pore volume are $151 \mathrm{~m}^{2} \mathrm{~g}^{-1}$ and $0.339 \mathrm{~cm}^{3} \mathrm{~g}^{-1}$ for pure $\mathrm{TiO}_{2}$, and $170 \mathrm{~m}^{2} \mathrm{~g}^{-1}$ and $0.457 \mathrm{~cm}^{3} \mathrm{~g}^{-1}$ for $12 \mathrm{wt} \% \mathrm{~h}-\mathrm{BN} / \mathrm{TiO}_{2}$, respectively. Compared with the pure $\mathrm{TiO}_{2}$, the SSA and pore volume of $12 \mathrm{wt} \% \mathrm{~h}-\mathrm{BN} / \mathrm{TiO}_{2}$ are improved by adding the flake hBN with high SSA, which is advantageous for improving
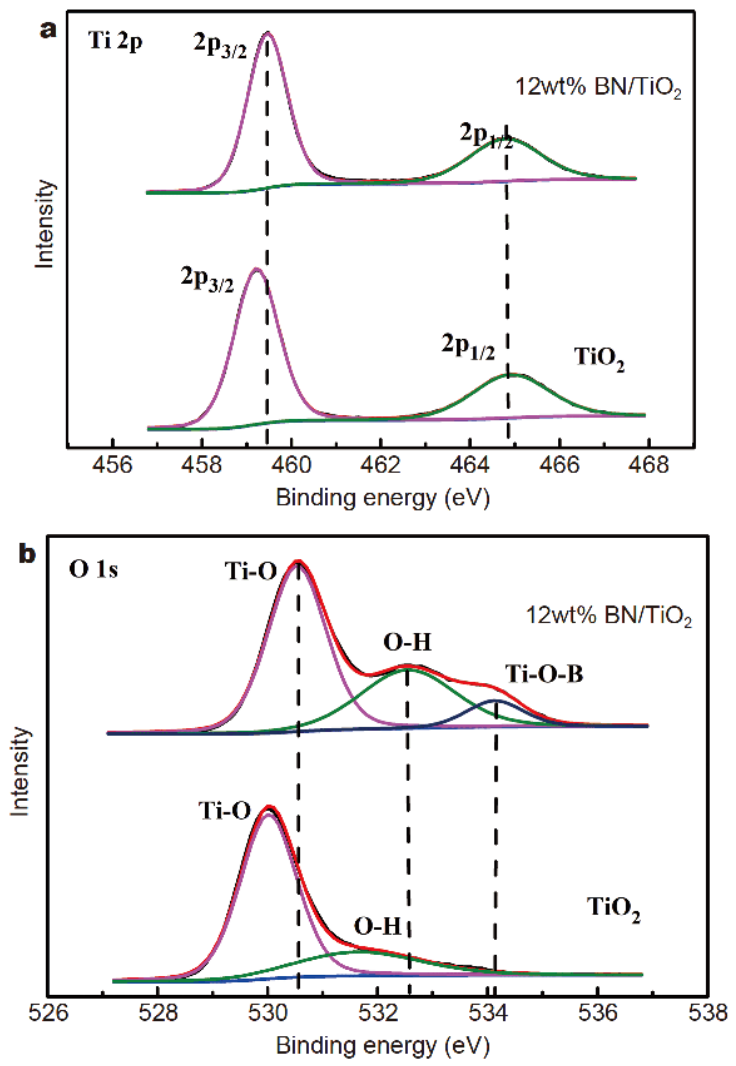

Figure 3 XPS spectra of as-prepared $\mathrm{TiO}_{2}$ and $12 \mathrm{wt} \% \mathrm{BN} / \mathrm{TiO}_{2}$ composite, (a) Ti 2p and (b) O1s.

the diffusion and adsorption capacity of organic dyes.

\section{Photocatalytic performance}

The photocatalytic performance of the h- $\mathrm{BN} / \mathrm{TiO}_{2}$ composites was evaluated by degrading $\mathrm{RhB}$ under visible light irradiation. Fig. 4a shows a comparison of degradation efficiency between $\mathrm{TiO}_{2}$ and $\mathrm{h}-\mathrm{BN} / \mathrm{TiO}_{2}$. Under visible light irradiation, the synthesized $\mathrm{TiO}_{2}$ displays a higher photocatalytic activity than commercial $\mathrm{TiO}_{2}$ (P25), which may be attributed to the morphology difference. Compared with the P25 nanoparticles, the flake $\mathrm{TiO}_{2}$ will offer more active sites for $\mathrm{RhB}$ degradation. It is well known that P25 can not absorb visible light, and thus the degradation is caused by self-photosensitized mechanism [32]. Meanwhile, it is noted that all the flake h$\mathrm{BN} / \mathrm{TiO}_{2}$ composites exhibit higher photocatalytic activity than the synthesized $\mathrm{TiO}_{2}$ and $\mathrm{P} 25$. The phenomenon is caused by the introduction of the flake h-BN with higher SSA which can greatly improve adsorption capacity and concentrate $\mathrm{RhB}$ molecules around $\mathrm{TiO}_{2}$. Among the composites, $12 \mathrm{wt} \% \mathrm{~h}-\mathrm{BN} / \mathrm{TiO}_{2}$ composite exhibits the highest photocatalytic activity and the degradation effi- 

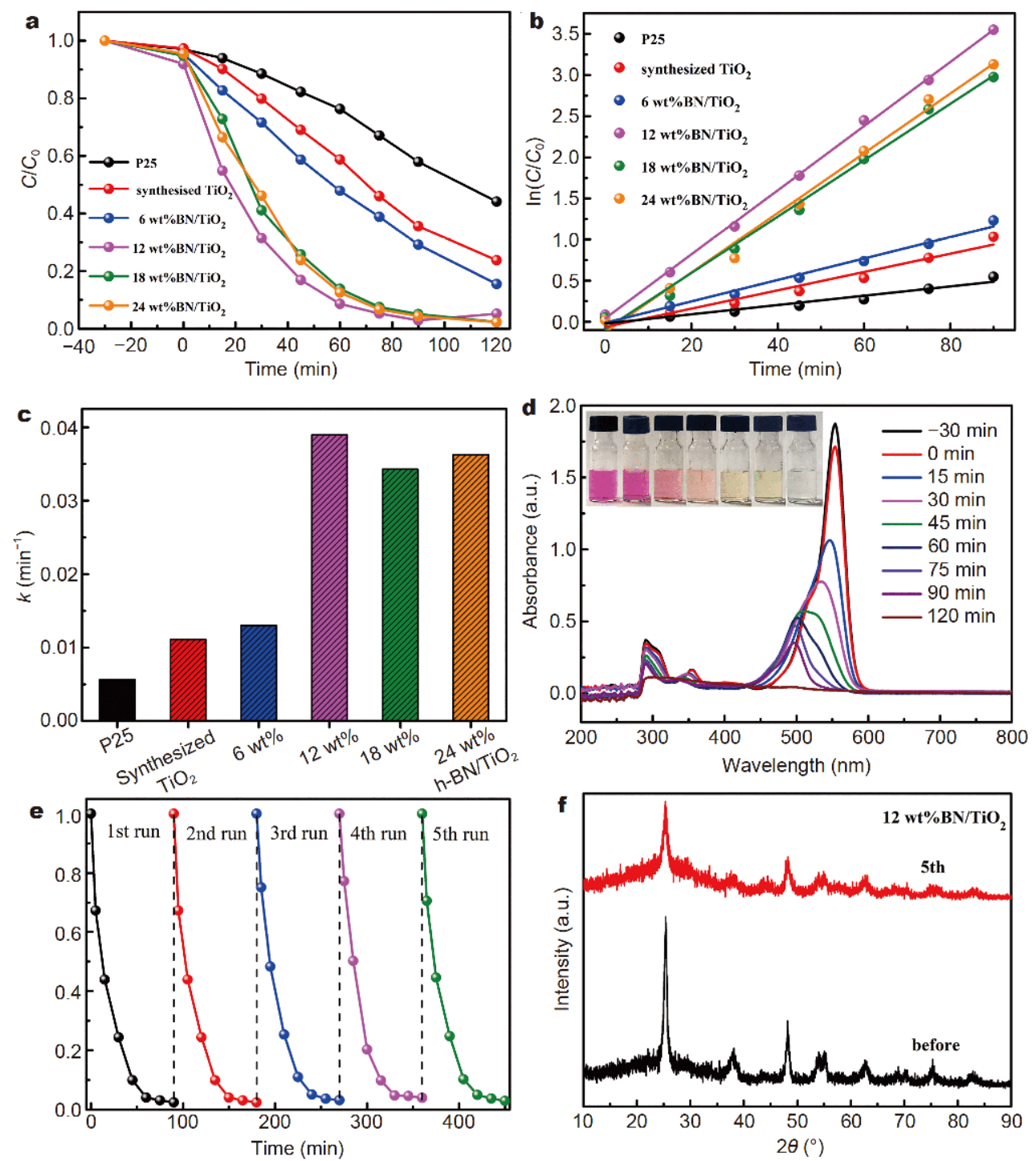

Figure 4 (a) Photodegradation rates of $\mathrm{RhB}$ and (b) kinetics of $\mathrm{RhB}$ degradation on $\mathrm{TiO}_{2}$ and $\mathrm{h}-\mathrm{BN} / \mathrm{TiO}_{2}$ samples and (c) the corresponding reaction rate constant $k$. (d) UV-vis absorption spectra and images (inset) of the aqueous $\mathrm{RhB}$ solution in the presence of $12 \mathrm{wt} \% \mathrm{~h}-\mathrm{BN} / \mathrm{TiO} \mathrm{O}_{2}$ at different time intervals. (e) Reusability property of $12 \mathrm{wt} \% \mathrm{~h}-\mathrm{BN} / \mathrm{TiO}_{2}$ composites on the photocatalytic degradation of RhB under visible light. (f) XRD patterns of the $12 \mathrm{wt} \% \mathrm{~h}-\mathrm{BN} / \mathrm{TiO}_{2}$ before and after the reusability experiment.

ciency is up to $95 \%$. For comparison, the physical mixture of $\mathrm{BN}$ and $\mathrm{TiO}_{2}$ (12 wt \% h-BN-TiO ${ }_{2}$ mixture) for $\mathrm{RhB}$ degradation was carried out. As shown in Fig. S4, about $40 \% \mathrm{RhB}$ is removed before light irradiation. However, only $13 \% \mathrm{RhB}$ is removed by using the same amount of $12 \mathrm{wt} \% \mathrm{~h}-\mathrm{BN} / \mathrm{TiO}_{2}$ composite. After irradiation, the composite exhibits higher photocatalytic activity than the mixture. The result indicates that immobilization of $\mathrm{TiO}_{2}$ on the surface of flake $\mathrm{h}-\mathrm{BN}$ is very effective for $\mathrm{RhB}$ degradation. Furthermore, phenol degradation was also performed to evaluate the photocatalytic activity and rule out the dye sensitization effect [33]. As shown in Fig. S5, $89 \%$ phenol is removed by $12 \mathrm{wt} \% \mathrm{~h}-\mathrm{BN} / \mathrm{TiO}_{2}$ after 150 min, which is much higher than $\mathrm{TiO}_{2}(17 \%)$. The superior photocatalytic activity of $12 \mathrm{wt} \% \mathrm{~h}-\mathrm{BN} / \mathrm{TiO}_{2}$ confirms that the photocatalytic activity under visible light is responsible for the degradation of phenol rather than the dye-sensitization effect.

The kinetic behavior of the composites for degradation of $\mathrm{RhB}$ was further investigated under visible light irradiation. Fig. $4 \mathrm{~b}$ shows the photocatalytic degradation of $\mathrm{RhB}$ following a pseudo first-order reaction: $\ln \left(C_{0} / C\right)=k t$, 
wherein $t, k, C_{0}$ and $C$ represent the reaction time, the apparent rate constant, the initial concentration and the concentration of RhB at given test time, respectively [34]. The kinetic constant $k$ was calculated and presented in Fig. 4c. The increasing order of the rate constants is: $12 \mathrm{wt} \% \mathrm{~h}-\mathrm{BN} / \mathrm{TiO}_{2}>24 \mathrm{wt} \% \mathrm{~h}-\mathrm{BN} / \mathrm{TiO}_{2}>18 \mathrm{wt} \% \mathrm{~h}-\mathrm{BN} /$ $\mathrm{TiO}_{2}>6 \mathrm{wt} \% \mathrm{~h}-\mathrm{BN} / \mathrm{TiO}_{2}>$ synthesized $\mathrm{TiO}_{2}>\mathrm{P} 25$. It is worth noting that the rate constant of $12 \mathrm{wt} \% \mathrm{~h}-\mathrm{BN} / \mathrm{TiO}_{2}$ is 3.5 and 6.9 times higher than that of the synthesized $\mathrm{TiO}_{2}$ and $\mathrm{P} 25$, respectively, which indicates photocatalytic activity of $\mathrm{TiO}_{2}$ is greatly improved by introducing appropriate amount of flake h-BN. Fig. 4d shows the UV-vis absorption spectra and images (inset) of the aqueous $\mathrm{RhB}$ solution in the presence of $12 \mathrm{wt} \% \mathrm{~h}-\mathrm{BN} / \mathrm{TiO}_{2}$ at different time intervals. It can be found that the maximum absorption wavelength displays a gradual blue shift from 554 to $498 \mathrm{~nm}$ with increasing irradiation time, which demonstrates both de-ethylation and oxidative degradation of $\mathrm{RhB}$ take place and the de-ethylation process is a stepwise manner [32]. The inset of Fig. $4 \mathrm{~d}$ shows the gradual fading of $\mathrm{RhB}$ color, indicating the $\mathrm{RhB}$ is decomposed completely. The present results are compared with those reported in the literature for dyes degradation (Table S1). It can be seen that the photocatalytic efficiency of the layered $\mathrm{h}-\mathrm{BN} / \mathrm{TiO}_{2}$ composite is comparable and even higher than other $\mathrm{BN}$-semiconductor systems, such as $\mathrm{BN} / \mathrm{Ag}_{3} \mathrm{VO}_{4}$ [10], $\mathrm{BN} / \mathrm{WO}_{3}$ [35], $\mathrm{BN} / \mathrm{BiOI}$ [36] and $\mathrm{BN} / \mathrm{g}-\mathrm{C}_{3} \mathrm{~N}_{4}$ [37]. Notably, benefiting from the unique flake-on-flake structure, the photocatalytic performance of flake h-BN/TiO ${ }_{2}$ composites is also superior to other $\mathrm{BN}-\mathrm{TiO}_{2}$ photocatalyst systems [28,38-43].

\section{Reusability and stability}

The stability and recyclability of $12 \mathrm{wt} \% \mathrm{~h}-\mathrm{BN} / \mathrm{TiO}_{2}$ were investigated by the same process as mentioned above. After each run of reaction, the photocatalyst was collected for continue using. From Fig. 4e, it can be seen that the removal percentage exhibits neglected change after five cycles, indicating the excellent reusability of $12 \mathrm{wt} \% \mathrm{~h}$ $\mathrm{BN} / \mathrm{TiO}_{2}$ composite. Fig. $4 \mathrm{f}$ shows the XRD patterns of the $12 \mathrm{wt} \% \mathrm{~h}-\mathrm{BN} / \mathrm{TiO}_{2}$ composite before and after the recyclability experiment. No obvious change of the phase can be found except for the lower characteristic diffraction peak intensity after test, indicating the good stability of the composite under irradiation.

\section{The photocatalytic mechanism}

The optical absorption spectra of the $\mathrm{TiO}_{2}$ and h-BN/ $\mathrm{TiO}_{2}$ composite are shown in Fig. S6. It can be seen that the absorption edge of $\mathrm{TiO}_{2}$ is determined to be $400 \mathrm{~nm}$.
Compared with the pure $\mathrm{TiO}_{2}$, h- $\mathrm{BN} / \mathrm{TiO}_{2}$ composites exhibit increased absorption ability in the visible light region, especially the $12 \mathrm{wt} \% \mathrm{BN} / \mathrm{TiO}_{2}$, which exhibits obvious red-shift of the absorption edge and has a long and weak absorption tail covering the whole visible light region. According to the plots of Kubelka-Munk remission function (i.e., relationship of $[\alpha h v]^{1 / 2} v s$. photon energy $\left(E_{\mathrm{g}}=h v\right)$, the calculated band gap values of the synthesized $\mathrm{TiO}_{2}$ is $3.1 \mathrm{eV}$, and the value can be decreased to $2.88 \mathrm{eV}$ for $12 \mathrm{wt} \% \mathrm{~h}-\mathrm{BN} / \mathrm{TiO}_{2}$, indicating a narrower band gap can be obtained, which is attributed to the energy arrangement caused by the heterojunctions between $\mathrm{TiO}_{2}$ nanoflakes and flake h-BN.

The PL emission spectra were measured with excitation light of $361 \mathrm{~nm}$ at room temperature. Generally, the lower PL intensity indicates lower recombination of charge carriers, resulting in higher photocatalytic activity [44]. Fig. 5a exhibits two main emission peaks centered at around 396 and $417 \mathrm{~nm}$, respectively. By comparison, $12 \mathrm{wt} \% \mathrm{~h}-\mathrm{BN} / \mathrm{TiO}_{2}$ exhibits the lowest intensity, demonstrating the lowest recombination rate of photogenerated electrons and holes, which is in accordance with the photocatalysis measurement (Fig. 4b). In addition, the photocurrent responses of $\mathrm{TiO}_{2}$ and $\mathrm{BN} / \mathrm{TiO}_{2}$ composites were studied as shown in Fig. 5 b. The h-BN/TiO ${ }_{2}$ composites exhibit a significantly enhanced photocurrent intensity compared with the $\mathrm{TiO}_{2}$, indicating much enhanced separation ability of electron-hole pairs after the introduction of $\mathrm{h}-\mathrm{BN}$, which results in much higher photocatalytic activity. EIS measurements were carried out as shown in Fig. $5 \mathrm{c}$. The $12 \mathrm{wt} \% \mathrm{~h}-\mathrm{BN} / \mathrm{TiO}_{2}$ exhibits the smallest arc radius, further indicating the lowest impedance, which can facilitate the interfacial chargetransfer process.

In order to further explore the photocatalytic mechanism of the composites, the trapping experiments were carried out to detect the main active species during the photocatalytic process. $1 \mathrm{mmol} \mathrm{L}^{-1}$ ethylenediaminetetraacetic acid disodium (EDTA-2Na), $2 \mathrm{mmol} \mathrm{L}^{-1}$ isopropanol (IPA) and $1 \mathrm{mmol} \mathrm{L}^{-1}$ benzoquinone (BQ) were chosen as scavengers for $\mathrm{h}^{+}, \cdot \mathrm{OH}$ and $\cdot \mathrm{O}_{2}^{-}$, respectively. From Fig. 5d, it can be seen that the effect of $\cdot \mathrm{OH}$ on the photocatalytic performance can be neglected, implying the $\cdot \mathrm{OH}$ does not contribute to the degradation of $\mathrm{RhB}$. However, the degradation rate significantly decreases when EDTA-2Na and BQ are added, indicating $\mathrm{h}^{+}$and . $\mathrm{O}_{2}{ }^{-}$play an important role in the photocatalytic process. In addition, electron spin resonance (ESR) spin-trap technique was also carried out to further detect the active species in the photocatalytic process as shown in Fig. 5e. 

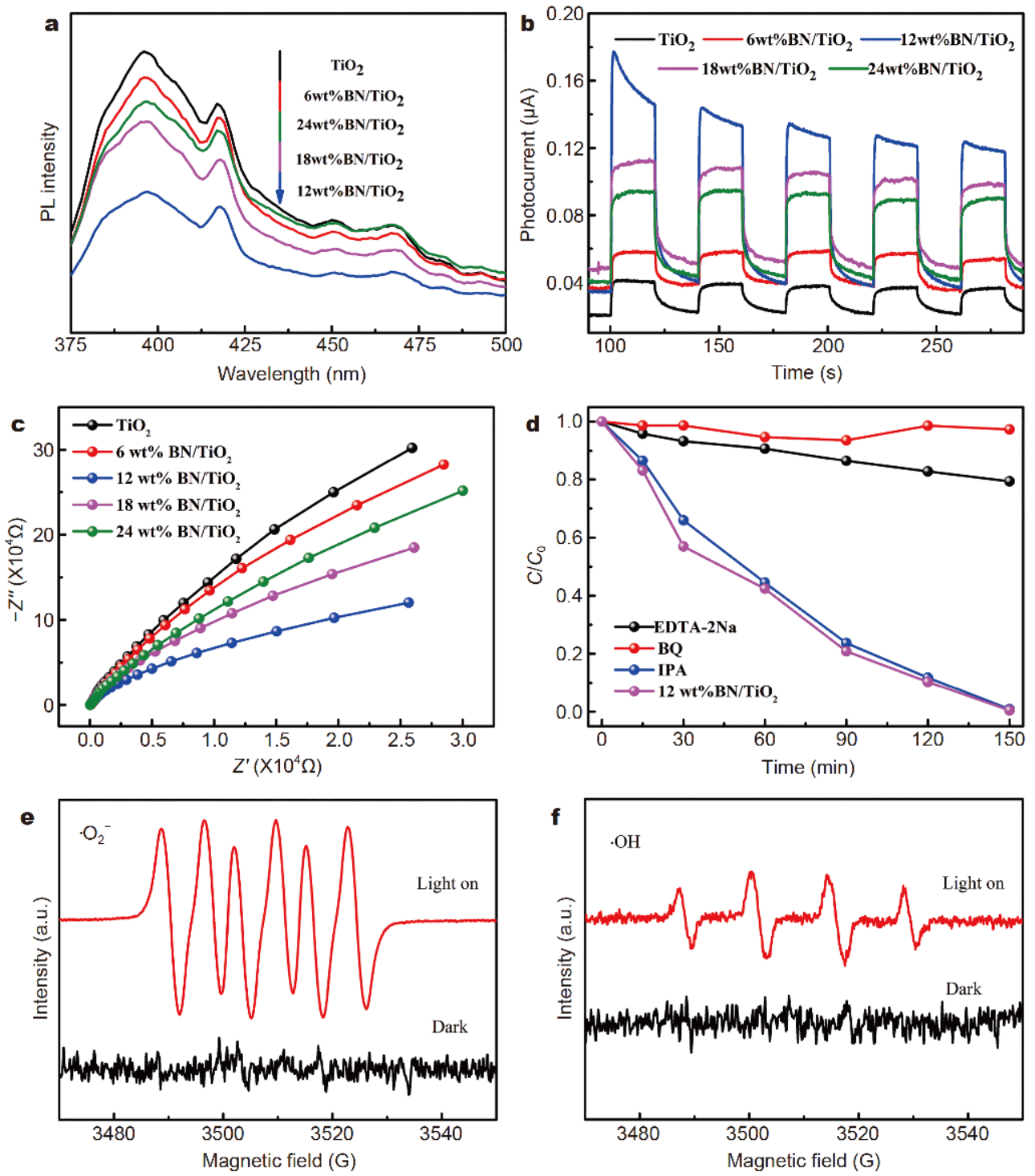

Figure 5 (a) PL emission spectra, (b) transient photocurrent responses, and (c) $\mathrm{EIS}$ of $\mathrm{TiO}_{2}$ and h- $\mathrm{BN} / \mathrm{TiO}{ }_{2}$ composites with different contents of flake h-BN. (d) Photocatalytic degradation of $\mathrm{RhB}$ on $12 \mathrm{wt} \% \mathrm{~h}-\mathrm{BN} / \mathrm{TiO}_{2}$ composites in the presence of different scavengers under visible light irradiation. DMPO spin-trapping ESR spectra in (e) methanol dispersion and (f) aqueous dispersion using 12 wt\% h-BN/TiO ${ }_{2}$ under visible light irradiation.

Six strong characteristic peaks of 5,5-dimethyl-1-pyrroline- $\mathrm{N}$-oxide (DMPO).$- \mathrm{O}_{2}^{-}$are observed in the methanol dispersion under visible light irradiation, while no signals can be found in the dark, indicating $\cdot \mathrm{O}_{2}{ }^{-}$radicals are indeed generated on the surface of irradiated $12 \mathrm{wt} \% \mathrm{~h}$ $\mathrm{BN} / \mathrm{TiO}_{2}$. Interestingly, the typical weaker characteristic peaks of DMPO-.OH (Fig. 5f) were also observed, indicating $\cdot \mathrm{OH}$ can be also produced. However, from the trapping experiment, we have found that the effect of $\cdot \mathrm{OH}$ on the photocatalytic performance can be neglected. Besides, the $\mathrm{VB}$ value of $\mathrm{TiO}_{2}$ after introduction of $\mathrm{BN}$ is not positive enough to oxidize $\mathrm{H}_{2} \mathrm{O}$ or $\mathrm{OH}^{-}$to $\cdot \mathrm{OH}(2.72 \mathrm{~V} v s$. normal hydrogen electrode (NHE)) [45]. Thus, the generation of $\cdot \mathrm{OH}$ may be attributed to the further reduction of $\cdot \mathrm{O}_{2}{ }^{-}$[46]. The results further prove that $\mathrm{RhB}$ can be degraded by $\mathrm{TiO}_{2}$ nanoflakes after the introduction of $\mathrm{h}$ BN under visible light. The mechanism of photocatalytic enhancement was investigated by employing DFT as shown in Fig. 6. The presence of the h-BN flake in the h$\mathrm{BN} / \mathrm{TiO}_{2}$ composite could effectively reduce the bandgap compared with the synthesized $\mathrm{TiO}_{2}$ (Fig. 6a and b), which is accordance with the result of the optical ab- 

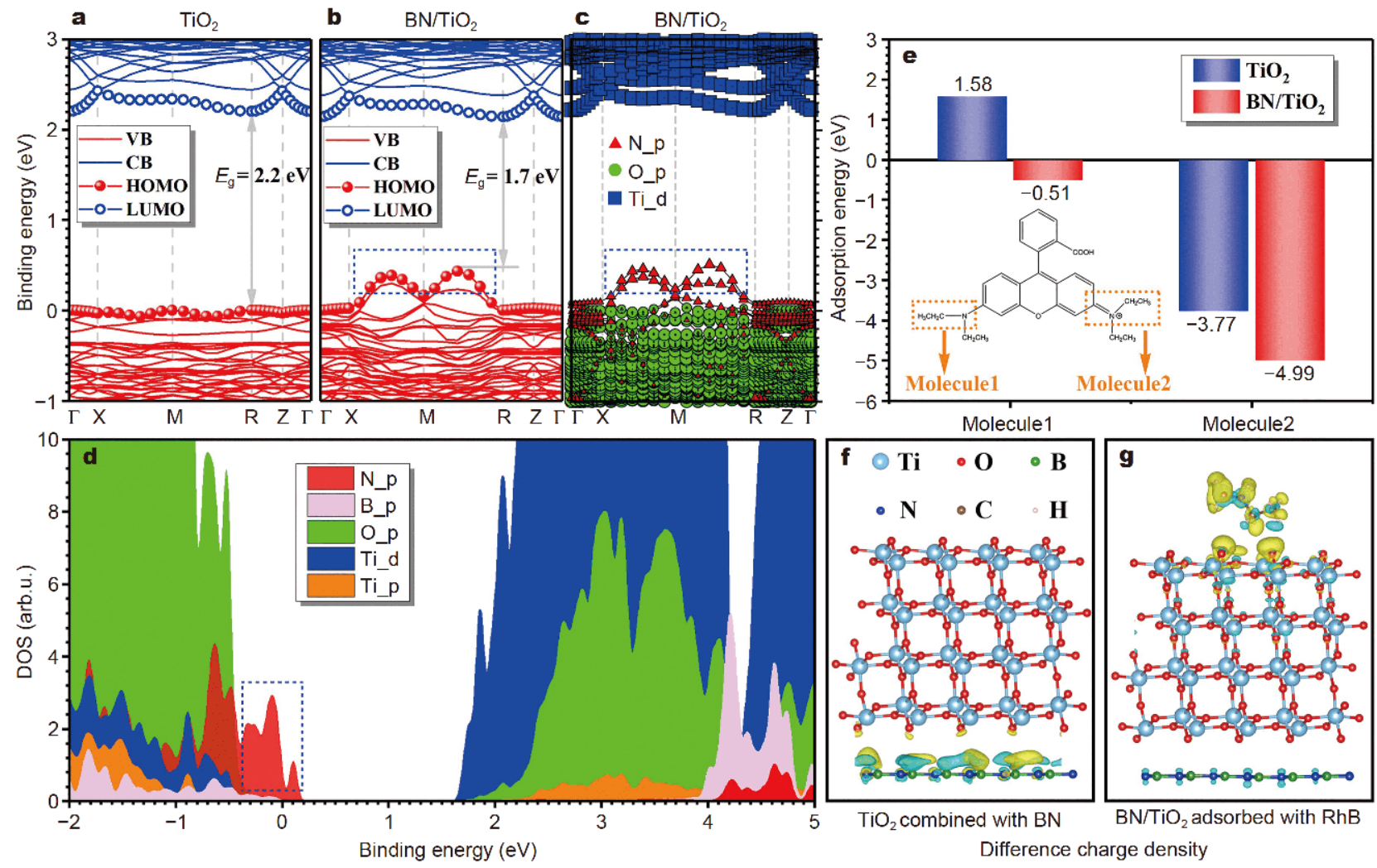

Figure 6 DFT calculation. (a, b) Band structures of $\mathrm{TiO}_{2}$ and h- $\mathrm{BN} / \mathrm{TiO}_{2}$, respectively. (c, d) Contribution of N, O and Ti orbitals on band structure and density of state, respectively. (e) The adsorption energy of two kinds of molecules with ethyl groups in $\mathrm{RhB}$ absorbed on $\mathrm{TiO}_{2}$ and h- $\mathrm{BN} / \mathrm{TiO}{ }_{2}$. (f, g) Difference charge densities of $\mathrm{TiO}_{2}$ combined with/without h-BN and h- $\mathrm{BN} / \mathrm{TiO}_{2}$ adsorbed with/without $\mathrm{RhB}$.

sorption spectra. The reduction of bandgap is caused by the upshift of VBM contributed by the N-p orbital of h$\mathrm{BN}$ (as shown in the two peaks in Fig. $6 \mathrm{~b}$ and c labeled by dashed boxes). Besides, CB and VB derive mainly from Ti-d and N-p orbitals, which could also be obtained from DOS in Fig. 6d. The state in VBM labeled by dashed box consists of N-p orbital, which lifts the VBM and reduces the bandgap. Furthermore, the band of the highest occupied molecular orbital (HOMO) of h- $\mathrm{BN} / \mathrm{TiO}_{2}$ is much more bent than that of $\mathrm{TiO}_{2}$ (Fig. 6a and b), clarifying that the hole of $\mathrm{h}-\mathrm{BN} / \mathrm{TiO}_{2}$ has much lighter effective mass than that of $\mathrm{TiO}_{2}$. The hole effective mass of $\mathrm{TiO}_{2}$ and $\mathrm{h}-\mathrm{BN} / \mathrm{TiO}_{2}$ is 0.57 and $0.12 m_{0}\left(m_{0}\right.$ is the rest mass of electron). Therefore, the addition of h-BN could increase the hole carrier mobility, which is beneficial for the oxidation of $\mathrm{RhB}$. In addition, two different parts of the RhB with ethyl group were chosen as the adsorbed molecules. Fig. 6e shows molecule 2 has more negative adsorption energy, i.e., easier to be adsorbed, and h- $\mathrm{BN} / \mathrm{TiO}_{2}$ has better adsorption ability than $\mathrm{TiO}_{2}$. Therefore, the flake $\mathrm{h}$-BN could increase the adsorption of $\mathrm{RhB}$ around the
$\mathrm{TiO}_{2}$ surface. The difference charge densities were also calculated to observe the charge transfer (Fig. 6f). There is large charge accumulation and charge depletion around $\mathrm{N}$ and $\mathrm{B}$ atoms, respectively, which will form the strong p-p coupling between the N-p and B-p orbitals, leading to the more bent HOMO (Fig. 6b). Meanwhile, there is also more or less charge accumulation around the $\mathrm{O}$ atoms close to h-BN, indicating the addition of h-BN can contribute electrons to $\mathrm{TiO}_{2}$. When molecule 2 is adsorbed on the surface of $\mathrm{h}-\mathrm{BN} / \mathrm{TiO}_{2}$, there is a large charge accumulation on the surface of $\mathrm{TiO}_{2}$, which leads to the electronic loss of molecule 2 and causes the de-ethylation process. DFT calculation further proves that the addition of h-BN into $\mathrm{TiO}_{2}$ can indeed enhance the photocatalytic performance.

Based on the above results, the possible mechanism of the h-BN/TiO ${ }_{2}$ is proposed as shown in Fig. 7. The CB and $\mathrm{VB}$ edge potentials of the synthesized $\mathrm{TiO}_{2}$ were theoretically speculated by means of atom's Mulliken electronegativity: $E_{\mathrm{CB}}=X-E_{\mathrm{C}}-0.5 E_{\mathrm{g}} ; \quad E_{\mathrm{VB}}=E_{\mathrm{g}}+E_{\mathrm{CB}}$, where $X$ represents the absolute electronegativity of the 
semiconductor; $E_{\mathrm{C}}$ and $E_{\mathrm{g}}$ are the energy of free electrons on hydrogen scale $(\sim 4.5 \mathrm{eV})$ and band gap, respectively [47]. The band edge potentials of CB and VB are -0.19 and $2.91 \mathrm{eV}$, respectively, as shown in Fig. 7. The redox potentials of $\mathrm{RhB}$ and excited $\mathrm{RhB}^{*}$ are 0.95 and $-1.42 \mathrm{eV}$ $v s$. NHE, respectively [48]. It is much easier for RhB to be oxidized at its excited state. Under illumination, due to the upshift of VB after the introduction of h-BN, electrons in $\mathrm{VB}$ can be excited to $\mathrm{CB}$, so holes are left on the VB. At the same time, RhB can be excited to form excited states $\left(\mathrm{RhB}^{*}\right)$, leading to electrons with injection from the adsorbed $\mathrm{RhB}^{*}$ species to the $\mathrm{TiO}_{2} \mathrm{CB}$ [49]. Due to the introduction of h-BN with high SSA, more adsorbed $\mathrm{RhB}^{*}$ species concentrate on the surface of $\mathrm{TiO}_{2}$. So, more electrons transfer to $\mathrm{CB}$ of $\mathrm{TiO}_{2}$. In addition, the reduction potential of $\mathrm{O}_{2} / \cdot \mathrm{O}_{2}^{-}$is $-0.046 \mathrm{eV} v \mathrm{~s}$. NHE [13]. Thus, the $\mathrm{CB}$ value of $\mathrm{TiO}_{2}$ is negative enough to reduce the adsorbed $\mathrm{O}_{2}$ to $\cdot \mathrm{O}_{2}^{-}$, which directly participate in the oxidation reaction. Importantly, both the flake-on-flake structure and the negatively charged h-BN will effectively promote $\mathrm{h}^{+}$transfer from the inner of $\mathrm{TiO}_{2}$ to its surface, and further improve the separation of photogenerated electron and hole pairs. Besides, Fig. 7 shows that the VB value of $\mathrm{TiO}_{2}$ is positive enough to oxidize $\mathrm{RhB}$. That is to say that the $\mathrm{h}-\mathrm{BN}$ flake works as a successive catalyst support material and plays a role of preventing the recombination of electrons and holes produced in $\mathrm{TiO}_{2}$ nanoflakes, following the synergetic catalytic effect of type II proposed in our previous work [50]. The possible reaction process for the decomposition of $\mathrm{RhB}$ is given in equations (3-9):

$$
\begin{aligned}
& \mathrm{TiO}_{2}+h v \rightarrow \mathrm{TiO}_{2}\left(\mathrm{e}^{-}+\mathrm{h}^{+}\right), \\
& \mathrm{RhB}+h v \rightarrow \mathrm{RhB}^{*}, \\
& \mathrm{RhB}^{*}+\mathrm{TiO}_{2} \rightarrow \mathrm{RhB}^{+}+\mathrm{TiO}_{2}\left(\mathrm{e}^{-}\right), \\
& \mathrm{TiO}_{2}\left(\mathrm{e}^{-}+\mathrm{h}^{+}\right)+\mathrm{BN} \rightarrow \mathrm{TiO}_{2}\left(\mathrm{e}^{-}\right)+\mathrm{BN}\left(\mathrm{h}^{+}\right), \\
& \mathrm{TiO}_{2}\left(\mathrm{e}^{-}\right)+\mathrm{O}_{2} \rightarrow \mathrm{TiO}_{2}+\cdot \mathrm{O}_{2}^{-}, \\
& \cdot \mathrm{O}_{2}^{-}+\mathrm{RhB}^{+} / \mathrm{RhB} \rightarrow \text { degradation } \ldots \text { products, } \\
& \mathrm{BN}\left(\mathrm{h}^{+}\right)+\mathrm{RhB}^{+} / \mathrm{RhB} \rightarrow \text { degradation } . . \text { products. }
\end{aligned}
$$

\section{CONCLUSIONS}

A novel $2 \mathrm{D} / 2 \mathrm{D} \mathrm{h}-\mathrm{BN} / \mathrm{TiO}_{2}$ composite photocatalyst with flake-on-flake nanostructure was successfully synthesized by a solvothermal method with in situ growth technology. As expected, the introduction of the flake h-BN can greatly improve the photocatalytic performance of the obtained composites. Especially for the $12 \mathrm{wt} \% \mathrm{~h}-\mathrm{BN} /$ $\mathrm{TiO}_{2}$ composite, a high photocatalytic efficiency up to

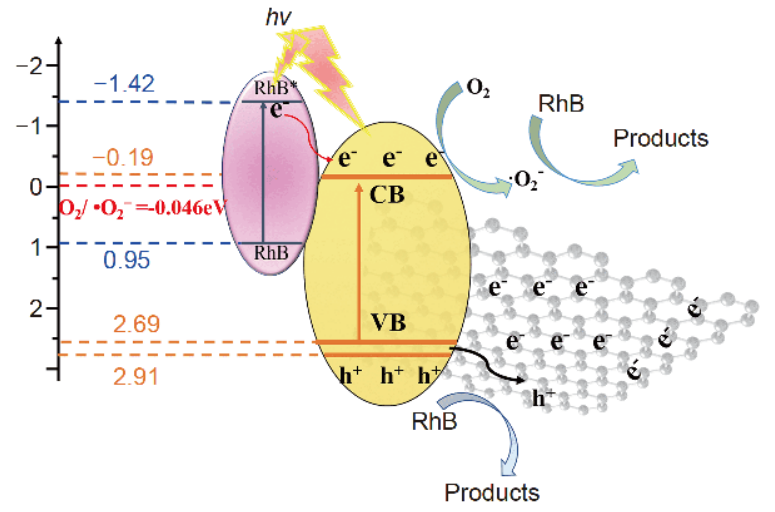

Figure 7 Photocatalytic degradation mechanism of h- $\mathrm{BN} / \mathrm{TiO}_{2}$ composite.

95\% for the degradation $\mathrm{RhB}$ under visible light has been obtained, and its rate constant is 3.5 and 6.9 times higher than that of the synthesized $\mathrm{TiO}_{2}$ and $\mathrm{P} 25$, respectively. Moreover, the $2 \mathrm{D} / 2 \mathrm{D}$ h- $\mathrm{BN} / \mathrm{TiO}_{2}$ composite exhibits excellent cycling stability and reusability. DFT calculation further proves that the addition of h- $\mathrm{BN}$ into $\mathrm{TiO}_{2}$ can indeed enhance the photocatalytic performance of composites. A synergetic catalytic mechanism has been formed on the heterojunctions between h-BN and $\mathrm{TiO}_{2}$ during the degradation of $\mathrm{RhB}$, in which the ability of visible light absorption is improved on one hand, and on the other hand the photosensitized $\mathrm{RhB}$ can increase the degradation rate of $\mathrm{RhB}$ by injecting electrons from the adsorbed $\mathrm{RhB}^{*}$ species to the $\mathrm{TiO}_{2} \mathrm{CB}$ to generate more active oxygen species $\cdot \mathrm{O}_{2}^{-}$. Meanwhile, not only the negatively charged h-BN improve the separation of photogenerated electron and hole pairs, but its larger specific surface area can also concentrate the $\mathrm{RhB}$ molecules around $\mathrm{TiO}_{2}$ to improve the kinetics. This work not only develops a new multifunctional h- $\mathrm{BN} / \mathrm{TiO}_{2}$ composite as photocatalysts under visible light irradiation, but also provides a new way to design advanced composite photocatalysts.

Received 21 June 2019; accepted 5 September 2019;

published online 25 October 2019

1 Shahabuddin S, Khanam R, Khalid M, et al. Synthesis of 2D boron nitride doped polyaniline hybrid nanocomposites for photocatalytic degradation of carcinogenic dyes from aqueous solution. Arabian J Chem, 2018, 11: 1000-1016

2 Liu W, Liu Z, Wang G, et al. Carbon coated $\mathrm{Au} / \mathrm{TiO}_{2}$ mesoporous microspheres: a novel selective photocatalyst. Sci China Mater, 2017, 60: 438-448

3 Tan Y, Liu M, Wei D. A simple green approach to synthesis of sub$100 \mathrm{~nm}$ carbon spheres as template for $\mathrm{TiO}_{2}$ hollow nanospheres with enhanced photocatalytic activities. Sci China Mater, 2018, 61: 
869-877

4 Fan $\mathrm{Y}, \mathrm{Hu} \mathrm{G}, \mathrm{Yu} \mathrm{S}$, et al. Recent advances in $\mathrm{TiO}_{2}$ nanoarrays/ graphene for water treatment and energy conversion/storage. Sci China Mater, 2019, 62: 325-340

5 Yin W, Bai S, Zhong Y, et al. Direct generation of fine $\mathrm{Bi}_{2} \mathrm{WO}_{6}$ nanocrystals on $\mathrm{g}-\mathrm{C}_{3} \mathrm{~N}_{4}$ nanosheets for enhanced photocatalytic activity. ChemNanoMat, 2016, 2: 732-738

6 Bera R, Kundu S, Patra A. 2D hybrid nanostructure of reduced graphene oxide-CdS nanosheet for enhanced photocatalysis. ACS Appl Mater Interfaces, 2015, 7: 13251-13259

7 Wang S, Luo $\mathrm{H}, \mathrm{Xu} \mathrm{X}$, et al. Enhanced organic dye removal of porous $\mathrm{BN}$ fibers supported $\mathrm{Ta}_{3} \mathrm{~N}_{5}$ nanoparticles under visible light irradiation. Surfs Interfaces, 2016, 5: 39-46

$8 \mathrm{Xu} \mathrm{H}, \mathrm{Wu} \mathrm{Z}$, Ding $\mathrm{M}$, et al. Microwave-assisted synthesis of flower-like $\mathrm{BN} / \mathrm{BiOCl}$ composites for photocatalytic $\mathrm{Cr}(\mathrm{VI})$ reduction upon visible-light irradiation. Mater Des, 2017, 114: 129138

9 Ide Y, Liu F, Zhang J, et al. Hybridization of Au nanoparticleloaded $\mathrm{TiO}_{2}$ with $\mathrm{BN}$ nanosheets for efficient solar-driven photocatalysis. J Mater Chem A, 2014, 2: 4150-4156

10 Lv X, Wang J, Yan Z, et al. Design of 3D h-BN architecture as $\mathrm{Ag}_{3} \mathrm{VO}_{4}$ enhanced photocatalysis stabilizer and promoter. $\mathrm{J} \mathrm{Mol}$ Catal A-Chem, 2016, 418-419: 146-153

11 Wang M, Li M, Xu L, et al. High yield synthesis of novel boron nitride submicro-boxes and their photocatalytic application under visible light irradiation. Catal Sci Technol, 2011, 1: 1159-1165

12 Wang J, Shen J, Fan D, et al. BN nanosheet: an efficient carriers transfer promoter and stabilizer to enhance the photocatalytic performance of $\mathrm{Ag}_{2} \mathrm{CO}_{3}$. Mater Lett, 2015, 147: 8-11

13 Ding S, Mao D, Yang S, et al. Graphene-analogue h-BN coupled Bi-rich $\mathrm{Bi}_{4} \mathrm{O}_{5} \mathrm{Br}_{2}$ layered microspheres for enhanced visible-light photocatalytic activity and mechanism insight. Appl Catal B-Environ, 2017, 210: 386-399

14 Ma F, Zhao G, Li C, et al. Fabrication of CdS/BNNSs nanocomposites with broadband solar absorption for efficient photocatalytic hydrogen evolution. CrystEngComm, 2016, 18: 631-637

15 Chen J, Zhu J, Da Z, et al. Improving the photocatalytic activity and stability of graphene-like BN/AgBr composites. Appl Surf Sci, 2014, 313: 1-9

16 Ji M, Xia J, Di J, et al. Graphene-like boron nitride induced accelerated charge transfer for boosting the photocatalytic behavior of $\mathrm{Bi}_{4} \mathrm{O}_{5} \mathrm{I}_{2}$ towards bisphenol A removal. Chem Eng J, 2018, 331: 355-363

17 Zhou C, Lai C, Zhang C, et al. Semiconductor/boron nitride composites: synthesis, properties, and photocatalysis applications. Appl Catal B-Environ, 2018, 238: 6-18

18 Zhang K, Fujitsuka M, Du Y, et al. 2D/2D heterostructured CdS/ $\mathrm{WS}_{2}$ with efficient charge separation improving $\mathrm{H}_{2}$ evolution under visible light irradiation. ACS Appl Mater Interfaces, 2018, 10: 20458-20466

19 Ye M, Gong J, Lai Y, et al. High-efficiency photoelectrocatalytic hydrogen generation enabled by palladium quantum dots-sensitized $\mathrm{TiO}_{2}$ nanotube arrays. J Am Chem Soc, 2012, 134: 1572015723

20 Li Q, Huo C, Yi K, et al. Preparation of flake hexagonal BN and its application in electrochemical detection of ascorbic acid, dopamine and uric acid. Sens Actuat B-Chem, 2018, 260: 346-356

$21 \mathrm{Fu} \mathrm{X}, \mathrm{Hu} \mathrm{Y}$, Zhang $\mathrm{T}$, et al. The role of ball milled h-BN in the enhanced photocatalytic activity: A study based on the model of ZnO. Appl Surf Sci, 2013, 280: 828-835
22 Perdew JP, Burke K, Ernzerhof M. Generalized gradient approximation made simple. Phys Rev Lett, 1996, 77: 3865-3868

23 Kresse G, Furthmüller J. Efficient iterative schemes for $a b$ initio total-energy calculations using a plane-wave basis set. Phys Rev B, 1996, 54: 11169-11186

24 Kresse G, Joubert D. From ultrasoft pseudopotentials to the projector augmented-wave method. Phys Rev B, 1999, 59: 1758-1775

25 Dudarev SL, Botton GA, Savrasov SY, et al. Electron-energy-loss spectra and the structural stability of nickel oxide: An LSDA+U study. Phys Rev B, 1998, 57: 1505-1509

26 Zhang J, Peng C, Wang H, et al. Identifying the role of photogenerated holes in photocatalytic methanol dissociation on rutile $\mathrm{TiO}_{2}$ (110). ACS Catal, 2017, 7: 2374-2380

$27 \mathrm{Hu} \mathrm{H}, \mathrm{Yu} \mathrm{L}$, Gao X, et al. Hierarchical tubular structures constructed from ultrathin $\mathrm{TiO}_{2}$ (B) nanosheets for highly reversible lithium storage. Energy Environ Sci, 2015, 8: 1480-1483

28 Liu D, Zhang M, Xie W, et al. Porous $\mathrm{BN} / \mathrm{TiO}_{2}$ hybrid nanosheets as highly efficient visible-light-driven photocatalysts. Appl Catal BEnviron, 2017, 207: 72-78

29 Zhang X, Lian G, Zhang S, et al. Boron nitride nanocarpets: controllable synthesis and their adsorption performance to organic pollutants. CrystEngComm, 2012, 14: 4670-4676

30 Chen D, Yang D, Wang Q, et al. Effects of boron doping on photocatalytic activity and microstructure of titanium dioxide nanoparticles. Ind Eng Chem Res, 2006, 45: 4110-4116

31 Li J, Huang Y, Liu Z, et al. Chemical activation of boron nitride fibers for improved cationic dye removal performance. J Mater Chem A, 2015, 3: 8185-8193

$32 \mathrm{Wu}$ T, Liu G, Zhao J, et al. Photoassisted degradation of dye pollutants. V. Self-photosensitized oxidative transformation of rhodamine $\mathrm{B}$ under visible light irradiation in aqueous $\mathrm{TiO}_{2}$ dispersions. J Phys Chem B, 1998, 102: 5845-5851

33 Long $\mathrm{M}$, Cai W, Cai J, et al. Efficient photocatalytic degradation of phenol over $\mathrm{Co}_{3} \mathrm{O}_{4} / \mathrm{BiVO}_{4}$ composite under visible light irradiation. J Phys Chem B, 2006, 110: 20211-20216

$34 \mathrm{Xu}$ J, Meng W, Zhang Y, et al. Photocatalytic degradation of tetrabromobisphenol A by mesoporous BiOBr: efficacy, products and pathway. Appl Catal B-Environ, 2011, 107: 355-362

$35 \mathrm{Xu} \mathrm{H}$, Liu L, Song Y, et al. BN nanosheets modified $\mathrm{WO}_{3}$ photocatalysts for enhancing photocatalytic properties under visible light irradiation. J Alloys Compd, 2016, 660: 48-54

36 Liu D, Jiang Z, Zhu C, et al. Graphene-analogue BN-modified microspherical BiOI photocatalysts driven by visible light. Dalton Trans, 2016, 45: 2505-2516

$37 \mathrm{Gu}$ J, Yan J, Chen Z, et al. Construction and preparation of novel 2D metal-free few-layer $\mathrm{BN}$ modified graphene-like $\mathrm{g}-\mathrm{C}_{3} \mathrm{~N}_{4}$ with enhanced photocatalytic performance. Dalton Trans, 2017, 46: 11250-11258

38 Nasr M, Viter R, Eid C, et al. Enhanced photocatalytic performance of novel electrospun $\mathrm{BN} / \mathrm{TiO}_{2}$ composite nanofibers. New J Chem, 2017, 41: 81-89

39 Singh B, kaur G, Singh $\mathrm{P}$, et al. Nanostructured $\mathrm{BN}-\mathrm{TiO}_{2}$ composite with ultra-high photocatalytic activity. New J Chem, 2017, 41: $11640-11646$

40 Si H, Lian G, Wang J, et al. Synthesis of few-atomic-layer BN hollow nanospheres and their applications as nanocontainers and catalyst support materials. ACS Appl Mater Interfaces, 2016, 8: 1578-1582

41 Xie W, Zhang M, Liu D, et al. Reactive yellow 161 decolorization by $\mathrm{TiO}_{2}$ /porous boron nitride nanosheet composites in cotton 
dyeing effluent. ACS Sustain Chem Eng, 2016, 5: 1392-1399

42 Fu X, Hu Y, Yang Y, et al. Ball milled h-BN: an efficient holes transfer promoter to enhance the photocatalytic performance of $\mathrm{TiO}_{2}$. J Hazard Mater, 2013, 244-245: 102-110

43 Liu D, Cui W, Lin J, et al. A novel $\mathrm{TiO}_{2-x} \mathrm{~N}_{x} / \mathrm{BN}$ composite photocatalyst: Synthesis, characterization and enhanced photocatalytic activity for rhodamine B degradation under visible light. Catal Commun, 2014, 57: 9-13

44 Zhou M, Yu J, Liu S, et al. Effects of calcination temperatures on photocatalytic activity of $\mathrm{SnO}_{2} / \mathrm{TiO}_{2}$ composite films prepared by an EPD method. J Hazard Mater, 2008, 154: 1141-1148

45 Li G, Wong KH, Zhang X, et al. Degradation of acid orange 7 using magnetic AgBr under visible light: the roles of oxidizing species. Chemosphere, 2009, 76: 1185-1191

46 Jiang L, Yuan X, Zeng G, et al. Metal-free efficient photocatalyst for stable visible-light photocatalytic degradation of refractory pollutant. Appl Catal B-Environ, 2018, 221: 715-725

47 Meng S, Ning X, Zhang T, et al. What is the transfer mechanism of photogenerated carriers for the nanocomposite photocatalyst $\mathrm{Ag}_{3} \mathrm{PO}_{4} / \mathrm{g}-\mathrm{C}_{3} \mathrm{~N}_{4}$, band-band transfer or a direct Z-scheme? Phys Chem Chem Phys, 2015, 17: 11577-11585

48 Fang Z, Li Q, Su L, et al. Efficient synergy of photocatalysis and adsorption of hexavalent chromium and rhodamine $\mathrm{B}$ over $\mathrm{Al}_{4} \mathrm{SiC}_{4} / \mathrm{rGO}$ hybrid photocatalyst under visible-light irradiation. Appl Catal B-Environ, 2019, 241: 548-560

49 Wang $\mathrm{Y}$, Wang $\mathrm{H}$, Xu A, et al. Facile synthesis of $\mathrm{Ag}_{3} \mathrm{PO}_{4}$ modified with GQDs composites with enhanced visible-light photocatalytic activity. J Mater Sci-Mater Electron, 2018, 29: 16691-16701

50 Shi J. On the synergetic catalytic effect in heterogeneous nanocomposite catalysts. Chem Rev, 2013, 113: 2139-2181

Acknowledgements This work was supported by the National Natural Science Foundation for Excellent Young Scholars of China (51522402), the National Postdoctoral Program for Innovative Talents (BX20180034), the Fundamental Research Funds for the Central Universities (FRF-TP-18-045A1), and China Postdoctoral Science Foundation (2018M641192).

Author contributions Hou X, Chen J, Liang T and Shi J supervised the experiment. Li Q and Yang T proposed the idea. Li Q designed and performed the experiment and wrote the paper. Fang $\mathrm{Z}$ contributed to the DFT calculations. Cui X and Hou X revised the paper. All authors contributed to the general discussion.

Conflict of interest The authors declare that they have no conflict of interest.

Supplementary information online version of the paper.

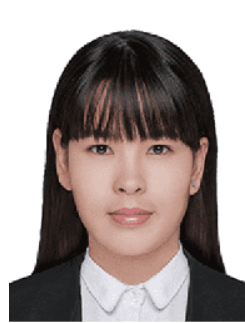

Qun Li is currently studying for her $\mathrm{PhD}$ degree in the University of Science and Technology Beijing, China. Her research focuses on the preparation of materials, electrochemical and adsorption application

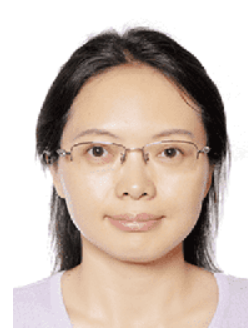

Xinmei Hou received her Bachelor's and Master's degree from Zhengzhou University, and got her $\mathrm{PhD}$ degree from the University of Science and Technology Beijing in 2009. Now she is a full professor of University of Science and Technology Beijing. Her research interests include the preparation of high performance ceramics, high temperature properties and functional applications in such fields of electrochemistry and environment.

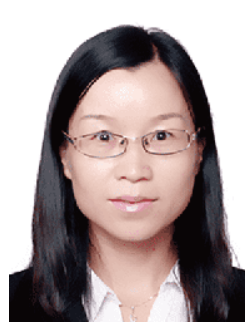

Xiangzhi Cui received her PhD degree in 2009 from Shanghai Institute of Ceramics, Chinese Academy of Sciences, and has been working at the institute since then. Her main research interest includes the structural design and synthesis of mesostructured nanocomposites, and the catalytic performances of the materials for applications in fuel cells and environmental protection.

\section{层状h-BN/TiO 2 异质结构的构建及其协同光催化 效应探讨}

李群 ${ }^{1}$, 侯新梅 ${ }^{*}$, 方志 $^{1}$, 杨涛 ${ }^{1}$, 陈俊红 ${ }^{2}$, 崔香枝 $^{3^{*}}$, 梁形祥 ${ }^{4}$, 施剑林 ${ }^{3}$

摘要 本文采用溶剂热法将纳米二氧化钛 $\left(\mathrm{TiO}_{2}\right)$ 片固定在六方氮 化硼(h-BN)片表面, 制备了一种新型层状h- $\mathrm{BN} / \mathrm{TiO}_{2}$ 复合光催化剂. 由于其独特的异质结构, 合成的复合材料对罗丹明 $\mathrm{B}(\mathrm{RhB})$ 降解的 光催化性能得到了提高, 其中 $12 \mathrm{wt} \% \mathrm{~h}-\mathrm{BN} / \mathrm{TiO}_{2}$ 的降解率分别比 合成的 $\mathrm{TiO}_{2}$ 和P25高3.5倍和6.9倍, 同时具有良好的循环稳定性. 此 外, 第一原理计算揭示了 $\mathrm{TiO}_{2}$ 与h-BN片之间的协同催化作用, 发现 h-BN的引入对h- $\mathrm{BN} / \mathrm{TiO}_{2}$ 复合材料的光催化性能有显著提高. 